\title{
Communications
}

\section{Site-Specific Modifications of the Cys-45 Residue in Human Dihydrolipoamide Dehydrogenase to Ser and Tyr}

\author{
Hakjung Kim \\ Deparment of Chemistry: College of Natural Science, Daegu Cniversitvi, Gvoungsan 712-714, Korea \\ E-mail: hjkimatalagu.ac.kr \\ Received Harch 15, 2007
}

Key Words : Dihỵ drolipoamide dehỵdrogenase. Pỵrridine nucleotide-disulfide oxidoreductase. Site-directed mutagenesis

Dihýdrolipoamide dehýdrogenase (E3) (dihyddrolipoamide: $\mathrm{NAD}^{+}$oxidoreductase: $\mathrm{EC}$ 1.8.1.4) along with glutathione reductase (GR). thioredoxin reductase (TR). mercuric reductase and trypanothione reductase, belongs to the pyridine nucleotide-disulfide oxidoreductase family. ${ }^{l}$ The catalytic mechanisms of pyridine nucleotide-disulfide oxidoreductases are similar and the active disulfide center plays a critical role in the catalyses of these enzymes. The Cys -45 of human E3 was suggested as one of two Cys residues which formed the active disulfide center of the enzyme." Sitedirected mutagenesis method has been a useful tool for the structure-function study of human E3 and other proteins. ${ }^{3-5}$

The following three questions have been investigated by site-directed mutations of the Cys-45 residue in human E3 to Ser and Tyr. First, how much is the activity of human E3

Table 1. Priners for the site-directed mutagenesis. The mismatched bases are underlined. Priner $A$ and Primer D are sense and antisense oligomers, respectively, which can be used to amplify the whole $E 3$ gene sequence. Primer $B$ is an anti-sense oligomer with a point mutation. Primer $\mathrm{C}$ is the corresponding sense oligomer of the primer $B$

\begin{tabular}{|c|c|}
\hline $\begin{array}{l}\text { Mutations } \\
\text { /Primers }\end{array}$ & Primer Sequences \\
\hline \multicolumn{2}{|c|}{ Cys-45 $\rightarrow$ Ser mutation } \\
\hline A & 5'-TTACGATATCCCAACGACCG-3' \\
\hline $\mathrm{B}$ & $\begin{array}{l}\text { 5'-GAAACACTTGGTGGAACATCCTTGAATGTTGG- } \\
\text { TTGTATT-3' }\end{array}$ \\
\hline $\mathrm{C}$ & $\begin{array}{l}\text { 5'-AATACAACCAACATTCAAGGATGTTCCACCAA- } \\
\text { GTGTTTC-3' }\end{array}$ \\
\hline $\mathrm{D}$ & 5'-GCCAAAACAAGCCAAGCTTGG-3' \\
\hline \multicolumn{2}{|c|}{ Cys-45 $\rightarrow$ Tyr mutation } \\
\hline A & 5'-TTACGATATCCCAACGACCG-3' \\
\hline $\mathrm{B}$ & $\begin{array}{l}\text { 5'-GAAACACTTGGTGGAACATACTTGAATGTTGG- } \\
\text { TTGTATT-3' }\end{array}$ \\
\hline $\mathrm{C}$ & $\begin{array}{l}\text { 5'-AATACAACCAACATTCAAGIATGTTCCACCAA- } \\
\text { GTGTTTC-3' }\end{array}$ \\
\hline $\mathrm{D}$ & 5'-GCCAAAACAAGCCAAGCTTGG-3' \\
\hline
\end{tabular}

affected by the mutation in the $\mathrm{Cys}-45$ residue? Second. are there any changes in the spectroscopic properties of human E3 due to the mutation? Third. is the production of human E3 in E. coll affected by the substitution of the Cys-45 residue with Tyr. a larger amino acid?

Site-directed mutagenesis and the constructions of the Ser45 mutant and Tyr-45 mutant expression vectors were performed by conmon procedures using the appropriate primers shown in Table 1. Mutations were confirmed by DNA sequencing. The expression and purification of the mutants were performed as described elsewhere. ${ }^{6}$ Purification steps were followed by SDS-polyacrylamide gel electrophoresis (data not shown). ${ }^{7}$ The gel showed that the Ser-45 mutant was highly purified. The purification of the Tyr-45 mutant was not successful. The amounts of the purified Tyr -45 mutant were too small and the mutant was too unstable to study. To examine the correctness of the expression vector component of the Tyr-45 mutant expression vector. human TR CDNA sequence was inserted instead of the Tyr-45 mutant sequence. Human TR mutant was expressed and purified properly (data not shomn). indicating that the expression vector component of the Tyr45 mutant expression vector did not have defects. The fundings suggest that the recombinant human E3 became too unstable to be easily obtained from $E$. coli when the Cys-45 was mutated to Tyr.

E3 activity of the Ser-45 mutant was detemined to be about $0.116 \mathrm{unit} / \mathrm{mg}$ at saturated substrate concentrations of $2 \mathrm{mM}$ dihydrolipoamide and $3 \mathrm{mM} \mathrm{NAD}{ }^{+}$at $37^{\circ} \mathrm{C}$. This value was about 5.270-fold lower than that of normal human E3 activity determined under the same conditions. Even though it was difficult to obtain the detailed kinetic parameters due to the extremely low activity of the mutant. this 5.270 -fold decrease in E3 activity of the Ser-45 mutant clearly indicated that the conservation of the $\mathrm{Cys}-45$ residue in human E3 was essential to the efficient catalytic function of the enzyme.

To examine any changes in the spectroscopic properties of the Ser-45 mutant. UV-visible absorption and fluore- 


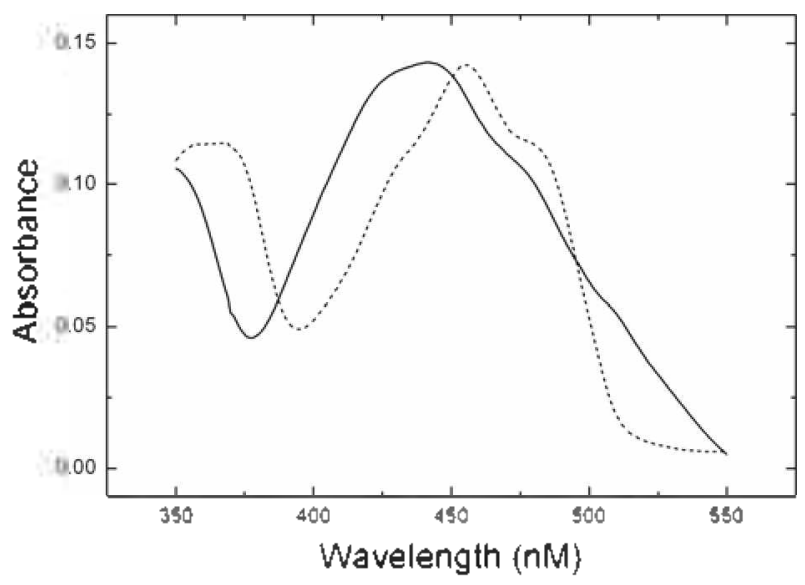

Figure 1. UV-visible spectra of the Ser-45 mutant $(15.8 \mu \mathrm{M}$, solid line) and wild-type $(12.9 \mu \mathrm{M}$, dotted line) human E $3 \mathrm{~s}$. The spectra were recorded using a SPECORD200 spectrophotometer and the data from $350 \mathrm{~nm}$ to $550 \mathrm{~nm}$ were transferred to an ASCII file and the spectra were drawn using the MicroCal Origin program.

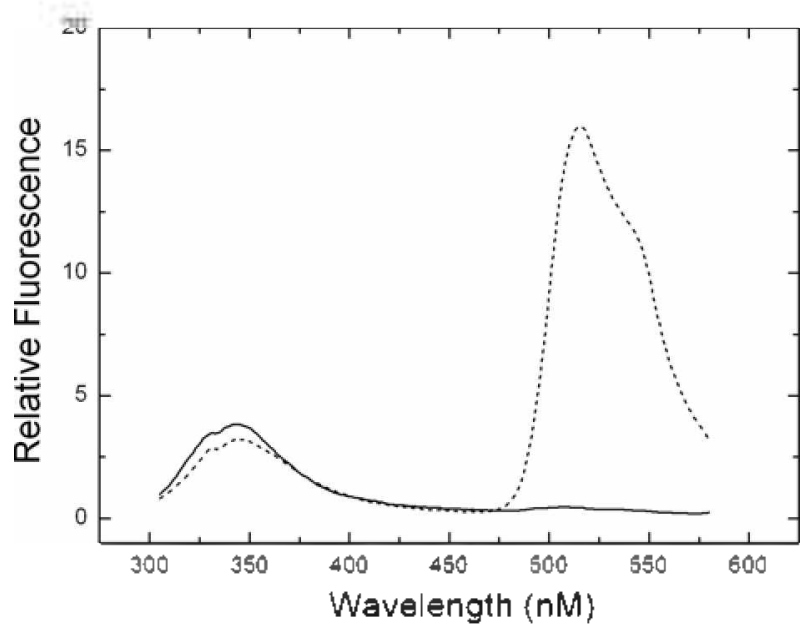

Figure 2. Flnorescence spectra of the Ser-45 mutant $(15.8, \mathrm{LM}$, solid line) and wild-type $(10.5 / \mathrm{M}$, dotted line $)$ human $\mathrm{E} 3$. Enzvmes were excited at $296 \mathrm{~nm}$ and the emissions were observed from $305 \mathrm{~mm}$ to $580 \mathrm{~mm}$. The data were transferred to an ASCII tile and the spectra were drawn using the MicroCal Origin progrann.

scence spectra were observed. Human E3 contains one FAD as a prosthetic group in each subunit. It therefore shows a characteristic UV-visible absorption spectrum of flavoproteins as shown in Figure 1 (dotted line). The overall shape of the Ser-45 mutant spectnum (solid line) is very different from that of nomal human E3 spectrum (dotted line) as shown in Figure 1. It was rather similar to that of the known spectrum of the reduced form of E3. where one of the negatively charged sulfur ions of the active disulfide center cysteines was thought to interact with $\mathrm{FAD}{ }^{1}$ It indicated that the substitution of the Cys-45 with Ser destroyed a disulfide bond between Cys-45 and Cys-50 of the active disulfide center in human E3. Then the free -SH group of the Cys-50 became deprotonated and the resulting negatively charged sulfur ion interacted with FAD. mimicking the reduced form of human E3. This result supports the known three-dimensional structure of human GR where the corresponding residue of the $\mathrm{Cys}-50$ is located more closely to $\mathrm{FAD}{ }^{8}$

More dramatic changes in the spectroscopic properties of the Ser-45 mutant came from the fluorescence study. As shown in Figure 2. two fluorescence emissions are observed for normal E3 (dotted line). The first emission from $305 \mathrm{~mm}$ to $400 \mathrm{~nm}$ is due to aromatic amino acids. mainly tryptophans. The second emission from $480 \mathrm{~nm}$ to over $550 \mathrm{~nm}$ is due to FAD. However, the second fluorescence emission of the Ser-45 mutant (solid line) totally disappears. This absence of the second fluorescence emission is probably due to the influence of the negatively charged sulfur ion of the Cys-50 interacting with $\mathrm{FAD}$. It can totally quench the second fluorescence emission or. perhaps, interferes with the fluorescence resonance energy transfer between tryptophan residues and $\mathrm{FAD}$ of the enzyme. The exact explanation could be obtained from the X-ray crystallograplic study of the Ser-45 mutant.

From these results. the following conclusions can be drawn. (1) The conservation of the Cys-45 residue is essential to the efficient catalytic function of human E3. (2) The UV-visible spectrum of the Ser-45 mutant is similar to that of the reduced form of the enzyme and the second fluorescence emission of the mutant disappears. (3) The mutation of the Cys-45 to Tyr in human E3 makes the enzyme too unstable to be easily obtained from $E$. coli.

Acknowledgements. The author thanks Dr. Mulchand S. Patel (State University of New York, Buffalo) for a generous gift of an $E$. coll XLI-Blue containing a human $\mathrm{E} 3$ expression vector. This research was supported in part by the Daegu University Research Grant. 2006.

\section{References}

1. Williams. C. H.. Ir. Enzzmes. 3rd ed: Boyer. P.. Ed.: Academic Press: 1976: $\mathrm{p} 89$

2. Jentoft, J. E.: Shoham, M.: Hurst, D; Patel, M. S. Proteins: Strtct. Funct. Genet 1992. 14.88.

3. Kim. H.: Patel, M. S. J. Biol. Chem 1992, 267, 5128 .

4. Kim. H. Bull Korean Chem. Soc. 2006. 27.819

5. Karim. M.: Shim. M.-Y.: Kim. J.: Choi. K.-T.: Kimn. T.-R.: Choi. J.-D: Yoon. M.-Y. Bull. Konean Chent. Soc. 2006.27 .549$.

6. Kim. H. J. Biochem. Mol. Biol. 2005. 38. 248

7. Kim. H. J. Biochem. Mlol. Biol. 2006. 39. 223.

8. Thieme. R.: Pai. E. F.: Schirmer, R. H.: Schulz. G. E. J. Mol Biol. 1981. 152.763 . 